\title{
Extensive multifocal branch duct IPMN of the pancreas after liver transplantation: is surgery justified?
}

\author{
Vittorio Branchi ${ }^{1,4}$, Philipp Lingohr ${ }^{1,4}$, Winfried A Willinek ${ }^{3,4}$, Alexander Semaan ${ }^{1,4}$, Hui Zhou ${ }^{2,4}$, Glen Kristiansen ${ }^{2,4}$, \\ Günter Klöppel ${ }^{5}$, Jörg C Kalff ${ }^{1,4}$, Nico Schäfer ${ }^{1,4}$ and Hanno Matthaei ${ }^{1,4^{*}}$
}

\begin{abstract}
Background: Cystic lesions of the pancreas resembling intraductal papillary mucinous neoplasms (IPMN) have been reported to develop in an increased rate following liver transplantation and immunosuppression. The cause for this possible association is thus far elusive.

Presentation of the case: We report on a 60-year-old male patient who developed an extensive multicystic change of the entire pancreas, suspicious for IPMN, under follow-up after liver transplantation for secondary sclerosing cholangitis. A total pancreaduodenectomy with splenectomy was performed. The postoperative histopathological assessment revealed a multifocal branch duct IPMN of the gastric subtype showing low-grade dysplasia.

Discussion: In the absence of evidence-based guidelines for the management of suspected IPMNs in liver transplant recipients, each patient's management should be discussed in detail.

Conclusion: Prospective studies may help to understand the disease and identify risk factors for malignant transformation in IPMNs after liver transplantation for treatment optimization.
\end{abstract}

Keywords: IPMN, Liver transplantation, Immunosuppression, Pancreatic cystic tumors

\section{Background}

Pancreatic cysts are increasingly diagnosed these days. Recent reports suggested a higher prevalence of IPMN-like cysts in patients after liver transplantation and subsequent immunosuppressive therapy. Since evidence-based guidelines are not established yet an ideal treatment is still controversially discussed sometimes causing a therapeutic dilemma.

\section{Case presentation}

A 60-year-old man was referred to our outpatient clinic because of persisting lower back pain and hyporexia. The patient was under follow-up after liver transplantation. Seven years prior to admission, he was treated at the intensive care unit for third degree burns covering

\footnotetext{
* Correspondence: hanno.matthaei@ukb.uni-bonn.de

'Department of Surgery, University Hospital of Bonn, Sigmund-Freud-Str. 25, 53127 Bonn, Germany

${ }^{4}$ Center for Integrated Oncology (CIO) Cologne-Bonn, Sigmund-Freud-Str. 25, 53127 Bonn, Germany

Full list of author information is available at the end of the article
}

approximately $30 \%$ of his body surface. During a complicated clinical course, he developed a severe secondary sclerosing cholangitis with biliary cirrhosis and was therefore listed for a liver transplantation. In November 2006, 8 months after the accident, he underwent whole living donor liver transplantation. Subsequently, the patient received a long-term immunosuppressive therapy with Tacrolimus, maintained at a blood concentration between 4.1 and $14.59 \mathrm{ng} / \mathrm{ml}$ and Mycophenolate $500 \mathrm{mg}$ every $8 \mathrm{~h}$. He regularly attended follow-up at our clinic and never suffered from any complications related to the immunosuppressive treatment regimen. Routine laboratory tests including blood count, liver function tests, bilirubin, and C-reactive protein were normal. He denied alcohol consumption and drug abuse.

Seven years after transplantation, a sonography performed for abdominal discomfort newly diagnosed multiple cystic lesions in the pancreatic head, body, and tail, the biggest lesion measuring $3.5 \mathrm{~cm}$ in maximum diameter. A magnetic resonance cholangiopancreatography 
(MRCP) confirmed the presence of multiple pancreatic cysts involving the entire gland (Figures 1 and 2). The lesions were morphologically compatible with a multifocal branch duct type intraductal papillary mucinous neoplasms (BD-IPMN). In order to obtain a biopsy for histopathological examination, an endoscopic retrograde cholangiopancreatography (ERCP) was carried out, which revealed a communication between the cysts and the dilated main pancreatic duct. Brush cytology showed papillary epithelium suspicious for IPMN. After thorough discussion in our interdisciplinary, weekly tumor board surgical resection was recommended. In November 2013, the patient underwent a total pancreatoduodenectomy and splenectomy. The histopathological assessment revealed a multifocal BD-IPMN of the gastric subtype with low-grade intraepithelial dysplasia (Figures 3, 4, and 5). No high-grade dysplasia or associated invasive cancer was detected on a multitude of sections from the entire specimen, which were independently assessed by three experienced pathologists (HZ, GK, GK). The postoperative course was uneventful, and the patient was discharged on the 14th postoperative day. Seven months after surgery, he presented in a favorable condition without any relevant complaints.

\section{Discussion}

This case reports the development of a multifocal branch duct type IPMN 7 years after liver transplantation and thus addresses the question regarding a causal relationship between liver transplantation and immunosuppression and the occurrence of cystic neoplasms in the pancreas. During the past few decades, the development of new immunosuppressive drugs and progress in surgical and perioperative treatment has led to a better prognosis after liver transplantation with more than half of those patients surviving for $>20$ years [1-3]. Development of neoplasms is a severe complication of long time immunosuppressive therapy [4]. The strong association between immunosuppression and cancer is nowadays well understood [5]. Several factors may contribute to carcinogenesis in liver transplant recipients including duration, type, and intensity of immunosuppression. As a consequence, cancer rates after liver transplantation are two- to threefold higher than in the age- and sexmatched population [6]. The most frequently observed neoplasm is post-transplant lymphoproliferative disorder (PTLD), usually observed relatively shortly after transplantation. Gastrointestinal cancers, in contrast, seem to be related to long-term immunosuppression [7]. In a recent large population-based study, the standardized incidence ratio (SIR) for de novo pancreatic cancer after liver transplantation was between 2.31 and 3.6 [8-11]. The literature about the onset of pancreatic cystic neoplasms after liver transplantation is, however, sparse. Girometti et al. showed that the prevalence of pancreatic cysts in patients after liver transplantation according to MRCP imaging was $59.6 \%$ [12]. Interestingly, $42.9 \%$ of the detected lesions showed features reminiscent of IPMN. These rates are considerably higher than in the general population although the prevalence of pancreatic cystic lesions in the literature varies considerably depending on the diagnostic method used. Reported numbers range from $0.2 \%$ on abdominal ultrasound [13], $2.6 \%$ on computed tomography $(\mathrm{CT})[14]$ to most representative $25 \%$ if considering postmortem necropsies in individuals without known pancreatic disease [15]. Thus

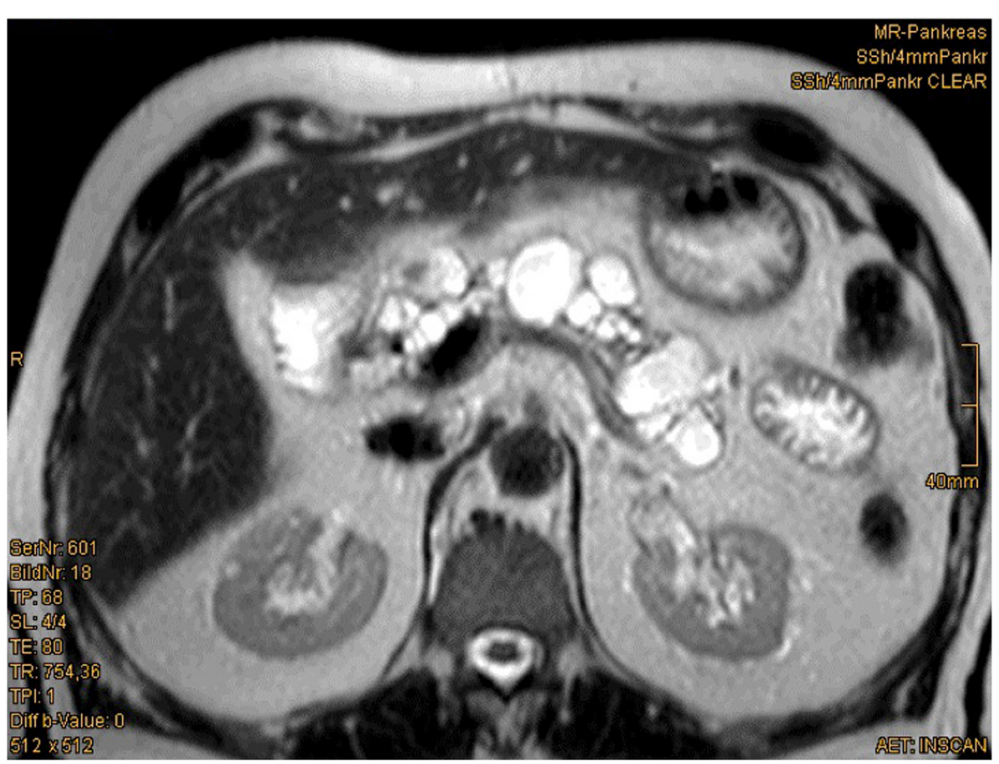

Figure 1 Magnetic resonance tomography (MRT) showing a multifocal cystic lesion of the pancreas involving the whole organ. 


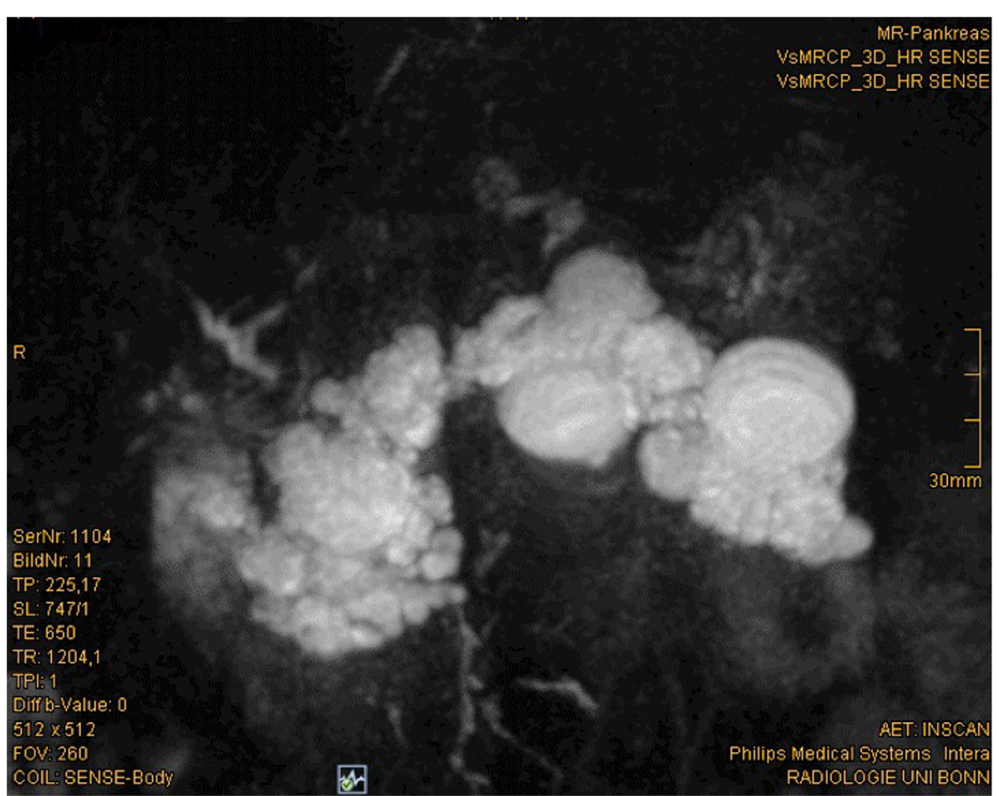

Figure 2 Magnetic resonance cholangiopancreatography (MRCP) with 3D reconstruction demonstrates the multicystic, grape-like appearance of the lesion.

far, it can only be speculated if the apparently higher incidence of pancreatic cystic lesions following liver transplantation is influenced by pre- or posttransplantation factors, such as preoperative pancreatic damage (for example, alcohol consumption or chronic pancreatitis) or postoperative immunosuppressive therapy. The clinical situation is certainly rare, and accordingly, the underlying pathomechanisms are poorly understood and no evidence-based treatment has been established. It is also unknown if IPMNs developing in patients after liver transplantation and immunosuppression have the same biologic behavior as 'sporadic' IPMNs.

There is an agreement that indication for abdominal surgery should be carefully evaluated in patients with liver transplants. Apart from possible severe adhesions due to prior major abdominal surgery, these patients have a high risk for infectious diseases with subsequent septic organ dysfunction. Nevertheless, in case of an IPMN with its potential to develop into a lethal invasive carcinoma, resection may be the only chance to prevent the patient from this deadly disease. The decision to

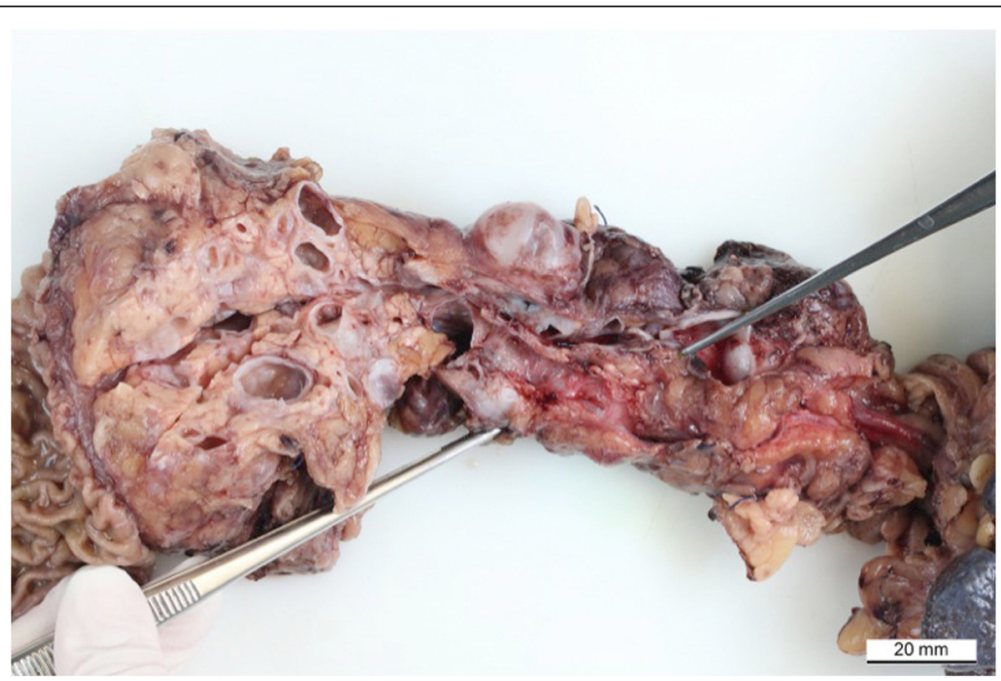

Figure 3 Formalin-fixed pancreas specimen that showed multiple cystic branch duct IPMNs while the main pancreatic duct appears only minimally dilated. 


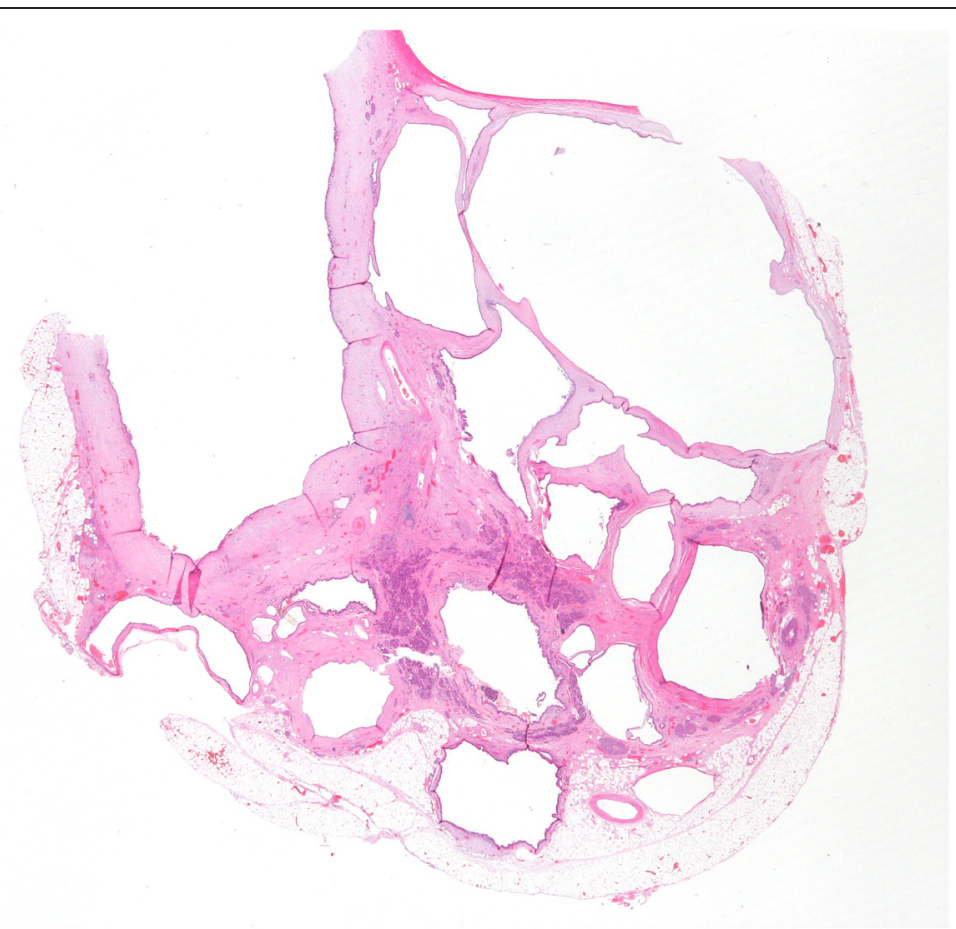

Figure 4 Hematoxylin and eosin staining showed IPMN-epithelium with merely low-grade dysplasia of the gastric epithelial subtype.

perform a total pancreatectomy in our patient was based on the following considerations: our patient had a multifocal cystic lesion involving the branch ducts of the entire pancreatic gland. Preoperative imaging also suspected a partial involvement of the main pancreatic duct, which appeared dilated up to $10 \mathrm{~mm}$, a 'worrisome feature' implying resection according to the Sendai consensus criteria. Additionally, our patient suffered from abdominal discomfort as potentially IPMN-associated symptom. With respect to the usually reduced life expectancy subsequent to liver transplantation, it is, however, questionable whether the Sendai criteria should be equally strictly applied in this patient population. According to the American Pancreatic Association (APA) international consensus guidelines in their latest version, surgical resection should be recommended in

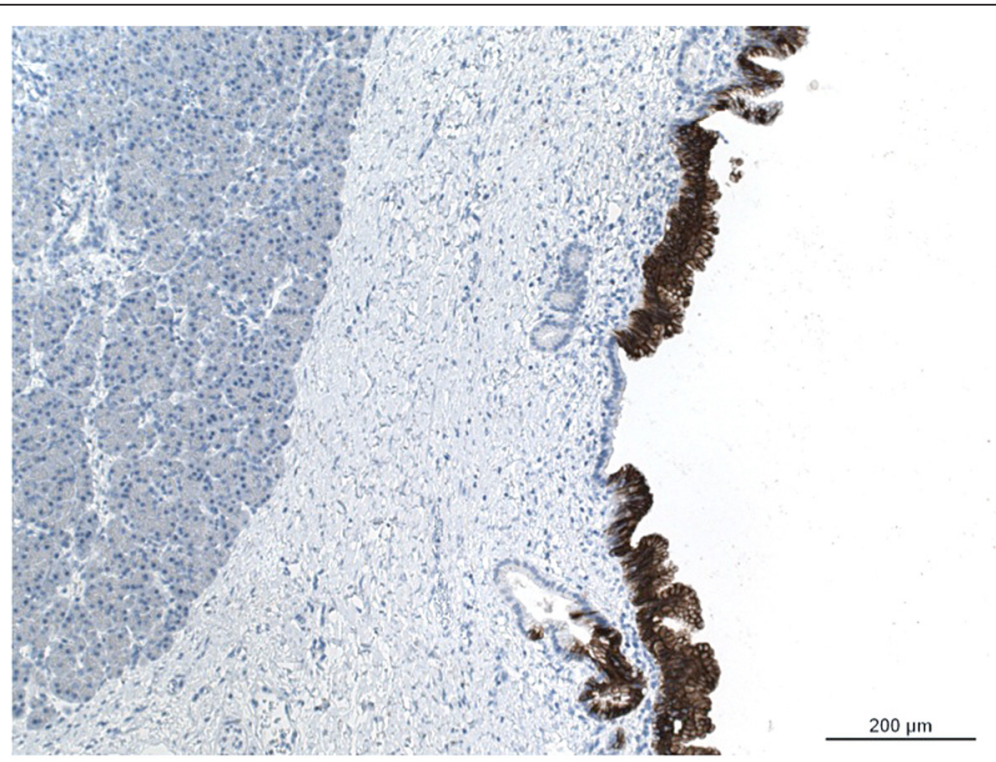

Figure 5 MUC5AC-immunoihstochemical staining of the epithelium confirmed the presence of gastric-type IPMN. 
patients with symptomatic cysts and in case of main pancreatic duct involvement, due to a higher incidence of invasive cancer $(43.1 \%$ versus $17.7 \%)$ [16,17]. In the literature, only a few reports are found on patients who underwent pancreatic surgery after liver transplantation while the number of liver transplant recipients undergoing major pancreatic surgery for IPMN seems even scarcer. A recent retrospective study including 3,196 patients who underwent liver transplantation at the University of Pittsburgh Medical Center during a period of 7.5 years demonstrated that $0.6 \%$ required pancreatic surgery in the postoperative course of transplantation [18]. Of those, merely one patient underwent distal pancreatosplenectomy for IPMN while six patients underwent pancreatectomy for malignant tumors. Stauffer et al. described a series of 17 patients who were referred to pancreatic surgery prior to, simultaneously, or after liver transplantation and reported only one patient who underwent a pancreaticoduodenectomy for de novo pancreatic ductal adenocarcinoma 3.8 years after liver transplantation [19]. Other authors reported single cases of new onset pancreatic cancer in patients after liver transplantation [20,21]. Del Chiaro et al. presented a patient who was successfully treated for a BD-IPMN through enucleation of the cyst 5 years post-liver transplantation [22]. Lennon et al. recently conducted a monocentric study to determine the risk of progression in patient with IPMNs after liver transplantation. In this retrospective analysis, 23 liver transplant recipients with BDIPMN were compared with a control group of 274 patients who did not undergo liver transplantation and with no history of immunosuppression [23]. Herein, $17.4 \%$ of the liver transplant patient and $16.4 \%$ in the control group developed high-risk features during the follow-up program. Therefore, no significant increase in the risk of progression of BD-IPMNs was found in patients after liver transplantation.

Prospective observation of liver transplant recipients focusing on cystic pancreatic changes in correlation with their clinical course will be essential to understand the conundrum of cyst formation in this patient cohort. Until we gather more information to develop evidencebased guidelines, we recommend careful individual management of every patient and to discuss any treatment in an experienced interdisciplinary tumor board along with Sendai recommendations.

\section{Conclusions}

Information on an increased occurrence of cystic lesions such as IPMN after liver transplantation and a possible etiological coherence is still sparse. From limited data available and our experience, there is no increased risk for progression towards invasive adenocarcinoma in this population. Thus, we recommend a critical and individual patient-by-patient evaluation in the therapeutic triage while recommendations from the Sendai consensus criteria should be respected. Patients should be treated in a tertiary referral center for hepatopancreatobiliary and transplant surgery/medicine.

\section{Consent}

Written informed consent was obtained from the patient for publication of this case report and any accompanying images. A copy of the written consent is available for review by the Editor-in-Chief of this journal.

\section{Abbreviations}

BD-IPMN: Branch duct IPMN; CT: Computed tomography; ERCP: Endoscopic retrograde cholangiopancreatography; IPMN: Intraductal papillary mucinous neoplasm; MRCP: Magnetic resonance cholangiopancreatography; PTLD: Post-transplant lymphoproliferative disorder; SIR: Standardized incidence ratio.

\section{Competing interests}

The authors declare that they have no competing interests.

\section{Authors' contributions}

VB mainly conceived of the case report, participated in its design and coordination, and helped significantly to draft the manuscript. PL participated in the clinical design of the manuscript and coordination and helped to draft the manuscript. WAW performed radiologic assessments and provided high-quality imaging material for the publication and helped to draft the manuscript. AS participated in its design and coordination and significantly helped to draft the manuscript. HZ, GK (Kristiansen) and GK (Klöppel) performed

histopathologic review and provided pathologic imaging material for the publication including molecular studies and helped to draft the manuscript. JCK conceived of the case report, participated in its design and coordination, and helped to draft the manuscript. NS: Conceived and co-supervised the case report, participated in its design and coordination, and helped to draft the manuscript. HM conceived and supervised the case report, participated in its design and coordination, and helped to draft the manuscript. All authors read and approved the final manuscript.

\section{Author details}

${ }^{1}$ Department of Surgery, University Hospital of Bonn, Sigmund-Freud-Str. 25, 53127 Bonn, Germany. ${ }^{2}$ Institute of Pathology, University Hospital of Bonn, Sigmund-Freud-Str. 25, 53127 Bonn, Germany. ${ }^{3}$ Department of Radiology, University Hospital of Bonn, Sigmund-Freud-Str. 25, 53127 Bonn, Germany. ${ }^{4}$ Center for Integrated Oncology (CIO) Cologne-Bonn, Sigmund-Freud-Str. 25, 53127 Bonn, Germany. ${ }^{5}$ Department of Pathology, Center for Pancreatic and Endocrine Tumors, Technical University Munich, Trogerstr. 18, 81675 Munich, Germany.

Received: 14 January 2015 Accepted: 5 March 2015

Published online: 19 March 2015

\section{References}

1. Jain A, Reyes J, Kashyap R, Dodson SF, Demetris a J, Ruppert K, et al. Long-term survival after liver transplantation in 4,000 consecutive patients at a single center. Ann Surg. 2000;232(4):490-500.

2. Duffy JP, Kao K, Ko CY, Farmer DG, MCDiarmid SV, Hong JC, et al. Long-term patient outcome and quality of life after liver transplantation: analysis of 20-year survivors. Ann Surg. 2010;252(4):652-61.

3. Schoening WN, Buescher N, Rademacher S, Andreou S, Kuehn A, Neuhaus A, et al. Twenty-year longitudinal follow-up after orthotopic liver transplantation: a single-center experience of 313 consecutive cases. Am J Transplant. 2013;13(9):2384-94.

4. Chandok N, Watt KD. Burden of de novo malignancy in the liver transplant recipient. Liver Transpl. 2012;18(11):1277-89.

5. Hanahan D, Weinberg RA. Hallmarks of cancer: the next generation. Cell. 2011;144(5):646-74. 
6. Rodríguez-Perálvarez M, De la Mata M, Burroughs AK. Liver transplantation: immunosuppression and oncology. Curr Opin Organ Transplant. 2014;19(3):253-60.

7. Herrero Jl, Lorenzo M, Quiroga J, Sangro B, Pardo F, Rotellar F, et al. De novo neoplasia after liver transplantation: an analysis of risk factors and influence on survival. Liver Transpl. 2005;11(1):89-97.

8. Åberg F, Pukkala E, Ho K, Sankila R, Isoniemi H. Risk of malignant neoplasms after liver transplantation: a population-based study. Liver Transpl. 2008; 14:1428-36

9. Jiang Y, Villeneuve PJ, Fenton SSA, Schaubel DE, Lilly L, Mao Y. Liver transplantation and subsequent risk of cancer: findings from a Canadian cohort study. Liver Transpl. 2008;14(11):1588-97.

10. Schrem H, Kurok M, Kaltenborn A, Vogel A, Walter U, Zachau L, et al. Incidence and long-term risk of de novo malignancies after liver transplantation with implications for prevention and detection. Liver Transpl. 2013;19(11):1252-61.

11. Krynitz B, Edgren G, Lindelöf B, Baecklund E, Brattström C, Wilczek H, et al. Risk of skin cancer and other malignancies in kidney, liver, heart and lung transplant recipients 1970 to 2008-a Swedish population-based study. Int J Cancer. 2013;132(6):1429-38.

12. Girometti R, Intini SG, Cereser L, Bazzocchi M, Como G, Del Pin M, et al. Incidental pancreatic cysts: a frequent finding in liver-transplanted patients as assessed by 3D T2-weighted turbo spin echo magnetic resonance cholangiopancreatography. JOP. 2009;10(5):507-14.

13. Ikeda M, Sato T, Morozumi A, Fujino MA, Yoda Y, Ochiai M, et al. Morphologic changes in the pancreas detected by screening ultrasonography in a mass survey, with special reference to main duct dilatation, cyst formation, and calcification. Pancreas. 1994;9(4):508-12.

14. Laffan TA, Horton KM, Klein AP, Berlanstein B, Siegelman SS, Kawamoto S, et al. Prevalence of unsuspected pancreatic cysts on MDCT. AJR Am J Roentgenol. 2008;191(3):802-7.

15. Kimura W, Nagai H, Kuroda A, Muto T, Esaki Y. Analysis of small cystic lesions of the pancreas. Int J Pancreatol. 1995;18(3):197-206.

16. Tanaka M, Chari S, Adsay V, Fernandez-del Castillo C, Falconi M, Shimizu M, et al. International consensus guidelines for management of intraductal papillary mucinous neoplasms and mucinous cystic neoplasms of the pancreas. Pancreatology. 2006;6(1-2):17-32.

17. Tanaka M, Fernández-del Castillo C, Adsay V, Chari S, Falconi M, Jang JY, et al. International consensus guidelines 2012 for the management of IPMN and MCN of the pancreas. Pancreatology. 2012;12(3):183-97.

18. Mejia J, Sucandy I, Steel J, Golas B, Humar A, Lee K, et al. Indications and outcomes of pancreatic surgery after liver transplantation. Clin Transplant. 2014;28(3):330-6. doi:10.1111/ctr.12317.

19. Stauffer JA, Steers JL, Bonatti H, Dougherty MK, Aranda-Michel J, Dickson RC, et al. Liver transplantation and pancreatic resection: a single-center experience and a review of the literature. Liver Transpl. 2009;15(12):1728-37.

20. Yoshizumi T, Shimada M, Soejima Y, Terashi T, Taketomi A, Maehara Y. Successful pylorus-preserving pancreaticoduodenectomy for a patient with carcinoma of the papilla Vater two years after living donor liver transplantation. Hepatogastroenterology. 2007;54(75):941-3.

21. Sutcliffe RP, Lam W, O'Sullivan A, Prachalias A, Rela M, Heaton N. Pancreaticoduodenectomy after liver transplantation in patients with primary sclerosing cholangitis complicated by distal pancreatobiliary malignancy. World J Surg. 2010;34(9):2128-32.

22. Del Chiaro M, Albiin N, Segersvärd R. Enucleation of branch duct-IPMN in a transplant patient. Pancreatology. 2013;13(3):312-3.

23. Marie Anne L, Victor D, Zaheer A, Ostovaneh MR, Jeh J, Law JK, et al. Liver transplant patients have similar risk of progression as sporadic patients with branch duct intraductal papillary mucinous neoplasms. Liver Transpl. 2014;20(12):1462-7.

\section{Submit your next manuscript to BioMed Central and take full advantage of:}

- Convenient online submission

- Thorough peer review

- No space constraints or color figure charges

- Immediate publication on acceptance

- Inclusion in PubMed, CAS, Scopus and Google Scholar

- Research which is freely available for redistribution

Submit your manuscript at www.biomedcentral.com/submit 